Egyptian

Orthodontic Journal

\title{
THE COMPARISON OF SOFT TISSUE PROFILE AFTER TREATMENT WITH FIXED FUNCTIONAL APPLIANCE, WITH DENTAL ANCHORAGE AND SKELETAL ANCHORAGE SYSTEMS
}

\author{
Wael M. Refai ${ }^{1}$
}

ABSTRACT:

Skeletal Class II due to mandibular retrusion is one of the major orthodontic problems. Nowadays, a great variety of non-compliance appliances are available to advance the mandible forwards. The study was carried out on 19 Class II Div 1 patients who were divided into two groups. The first group was treated using Twin Force Bite Corrector (TFBC) while the second Group was treated with Twin Force Bite Corrector with skeletal anchorage using mini-plates. Pre-treatment and 3 months post-treatment cephalograms were obtained. The changes in the soft tissue profile were inspected. The results showed significant changes in soft tissue measurements after placing TFBC. In addition, there was a significant advancement in soft tissue pogonion in the second group due to the more mandibular advancement.

Key words: soft tissue profile, fixed functional appliances, skeletal anchorage

\section{INTRODUCTION}

Orthodontic treatment in patients with limited compliance can result in longer treatment time, frustration for the patient, additional stress for the orthodontist and staff. This is why a lot of effort has been directed over the years to developing noncompliance techniques. The latter was important in treating uncooperative Class II patients.

1- Assistant Professor of Orthodontics, Minia University 
The data on the degree of compliance are rather limited. Microelectronic monitoring showed an average of 7.65 hours per day of functional appliance wear which was only a 50-60\% fulfillment of the wearing instructions, decreasing to below 35\% at the sixth appointment (Sahm, Bartsch and Witt 1990a). ${ }^{(1)}$

Various aspects of compliance prediction were discussed in detail in a published review by (Sergi and Zentner 2000) ${ }^{(2)}$. However, no single parameter or a clearly defined parameter group has so far been identified as a reliable predictor (Nanda and Kierl .1992. Sergi and Zentner 2000). ${ }^{(3,2)}$

Noncompliance treatment modalities are not solely indicated in patients with minimal compliance but also can be applied in compliant patients. They can be used in patients with almost prepubertal growth, at the early phase of permanent dentition, and when second maxillary molars have already erupted. Using these modalities, the treatment procedures are better controlled by orthodontist and therefore more predictable results can be expected.

Class II division 1 malocclusion is considered to be the most frequent problem encountered in orthodontics. This was reported by many previous studies (Goldstein and Stanton1936 ${ }^{(4)}$ Fisk1960 $^{(5)}$, Ast, Carlos, and Cons $1965^{(6)}$. McNamara $(1981)^{(7)}$ considered that skeletal mandibular retrusion was the most common characteristic in Class II division 1 malocclusion.

The main problem of treating skeletal Class II with fixed functional appliances is the difficulty in controlling the proclination of the lower incisors and the advancement of all the mandibular teeth. These can compromise the mandibular advancement and the final esthetic result.

Application of orthodontic tooth movement using skeletal anchorage (SAS) offered capabilities which couldn't yet be obtained by any available appliance. With screws, pins, or some other readily removable implants anchored to the jaws, forces are applied to produce tooth movement in any direction without detrimental reciprocal forces. Accordingly, orthopedic forces are applied directly to the jaws through skeletal anchorage rather than through tooth borne anchorage. 
Egyptian

Orthodontic Journal

Although many researches had been conducted to investigate the efficacy of SAS from the points of efficiency, changes in the skeletal and dental structures, changes in the soft tissues were not given the importance they deserve. Accordingly, this study was conducted to highlight this aim.

\section{MATERIAL AND METHODS}

The study included 19 subjects who suffered from Class II malocclusion. Their age ranged between 11 and 14 years. The malocclusion was due to mandibular retrusion. The surgical part was explained to the patients and their parents before starting the treatment. Patients were divided into two groups: I and II. For group I, the Twin Force Bite Corrector ${ }^{*}$ (Double lock version) (TFBC) was used. In group II, TFBC with skeletal anchorage system (SAS) was used.

Orthodontic treatment with fixed appliance was carried out after taking all pre-treatment records. A transpalatal arch was constructed at the beginning then leveling and alignment were started. Treatment was proceeded till reaching heavy rectangular arch wire in both arches. A lateral cephalogram (T1) was taken before inserting the appliance. In addition, archwires were cinched back.

Group I (TFBC group): The TFBC was selected according to the distance measured between the distal part of the lower canine bracket and mesial end of the tube of the upper first molar band. Measurement was taken when the patient was biting in an edge to edge relationship. The appliance was attached by securing the clamp around the archwire. (Fig 1)

Patients were then rechecked after one week for any complaints and then once every two weeks. At each visit, the appliance was removed from the mandibular attachment on both sides to check molar and canine relationships. When molar and canine Class I relationships were obtained, the appliance was removed.

For settling the occlusion, the patients were instructed to wear $1 / 4$ inch medium intermaxillary elastics from the maxillary canines and first premolars to the mandibular canines and first molars. (Fig 2) Elastic were worn for 3 months. Thereafter, a second lateral cephalogram (T2) was taken.

*Ortho Organizers, San Marcos, California

Volume 40 - December 2011 


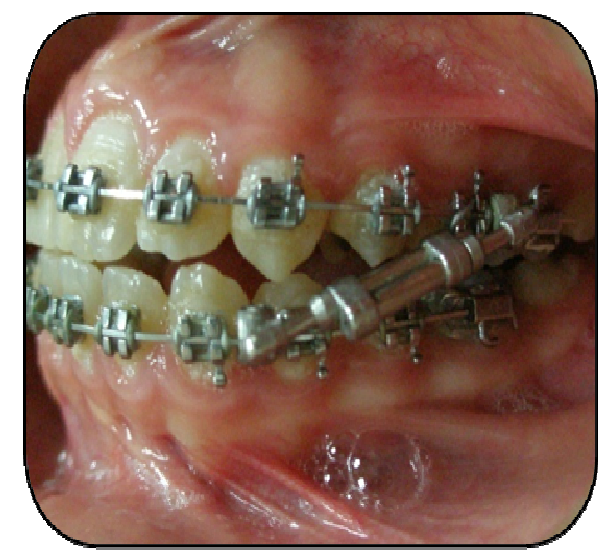

Fig 1: side view showing TFBC \& edge to edge occlusion

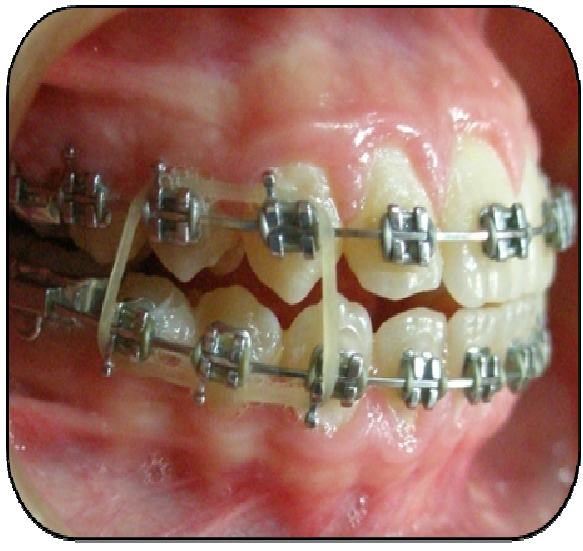

Fig 2: Elastics worn to settle the occlusion

Especially designed titanium miniplates (Fig 3) were used for skeletal anchorage. A $35 \mathrm{~mm}$ long straight 3-hole miniplate was used. The plate consists of three parts:

1- The endossous part: (15 mm length, $0.7 \mathrm{~mm}$ thickness) with the 3 hole where the miniscrews were screwed in the bone

2- The connector part: (16 mm length, $1.4 \mathrm{~mm}$ thickness) round and smooth connecting between endossous sector and attachment sector.

3- The attachment part: (4 $\mathrm{mm}$ length, $0.5 \mathrm{~mm}$ thickness) the part that the TFBC is attached to.

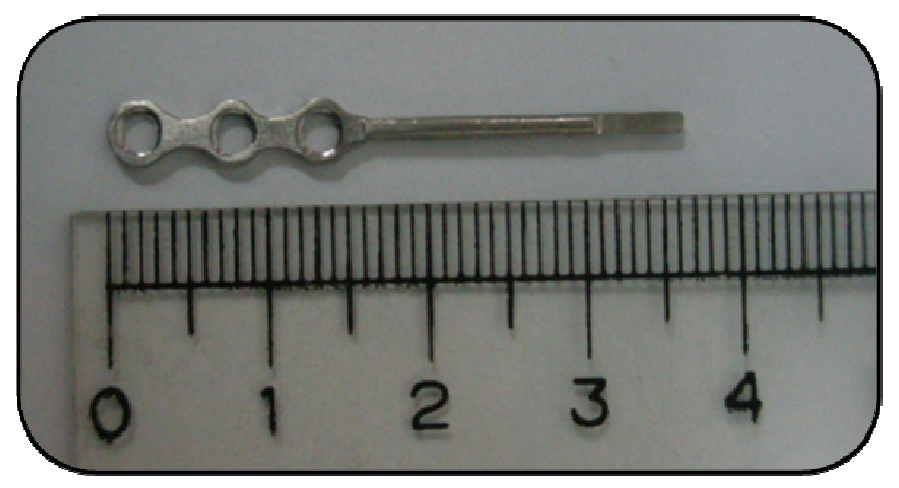

Fig 3: Especially designed mini plate 
Egyptian

Orthodontic Journal

\section{Miniscrew}

Self-cutting and $9 \mathrm{~mm}$ long miniscrews were used. They were made of pure titanium

\section{Surgical Procedure:}

After route block anesthesia, an incision was made. After folding back the mucous membrane and periosteum flap, the miniplate was bent and adjusted to the bone surface. This was done to achieve maximum bone contact.

The neck section was adjusted so that a spacing of $1.5-2 \mathrm{~mm}$ was made between the attachment sector and the marginal mucous membrane. The miniplate was placed so that the bend layed directly at the edge of the wound-line. All screws were placed with a hand driver (blade \#25-483-97, handle \#25-402-99, KLS Martin) after pilot-hole drilling as the manufacturer recommended.

The miniplate was then attached with three microscrews $9 \mathrm{~mm}$ length, (Fig 4) and the wound was flushed out with a sodium chloride solution and closed with two to four interrupted over-and-over sutures. (Fig 5) They were removed on the seventh postoperative day. The last part on the plate is allowed to project transmucosally into the buccal vestibule. (Fig 6) The TFBC was attached to the last part on the miniplate. (Fig 7-10)

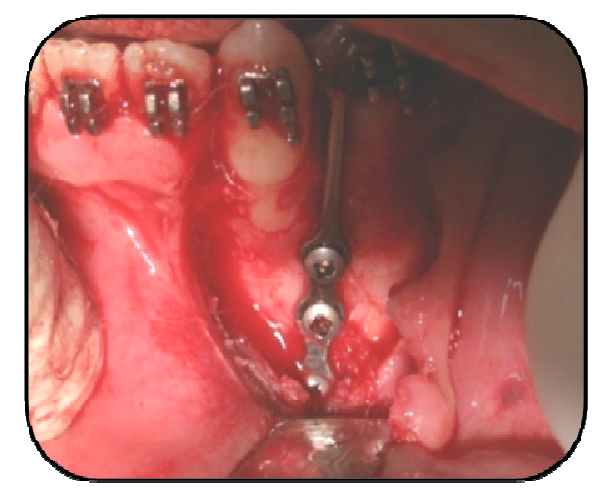

Fig 4: Fixation of the miniplate

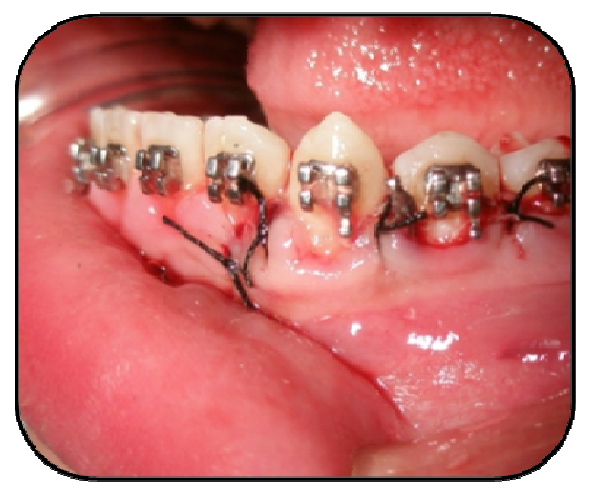

Fig 5: Sutures over the miniplate

Volume 40-December 2011 


\section{Egyptian}

Orthodontic Journal

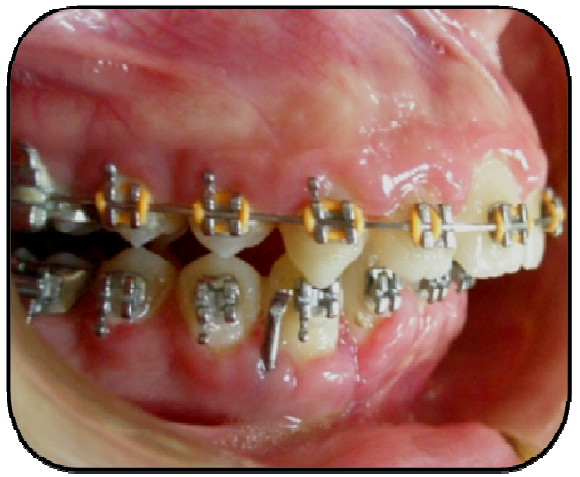

Fig 6: Miniplate projecting transmucosally

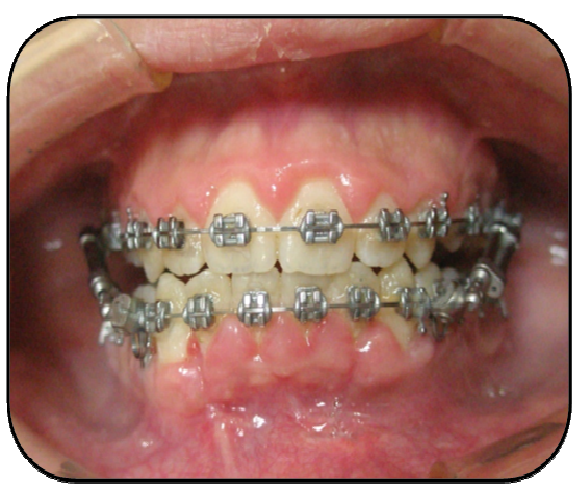

Fig 8: Frontal view: After fixing TFBC over the miniplate

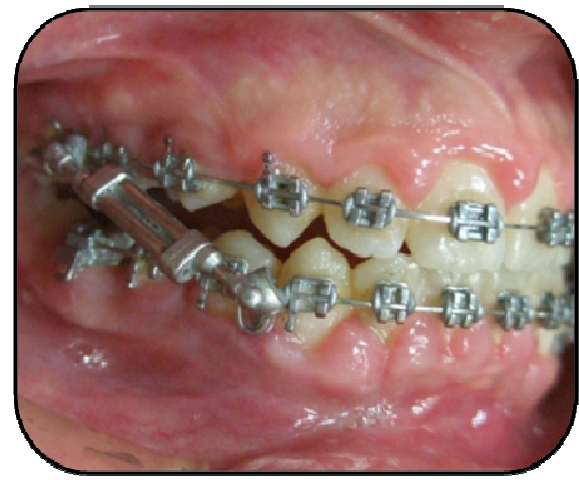

Fig 7: TFBC fixed over the miniplate

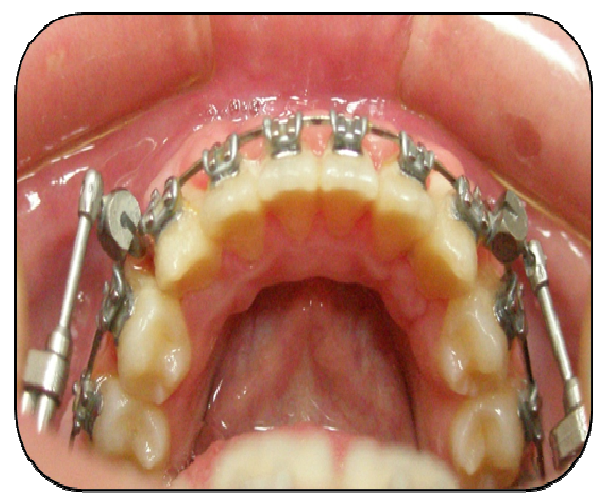

Fig 9: Lower occlusal view: After fixing TFBC over the miniplate

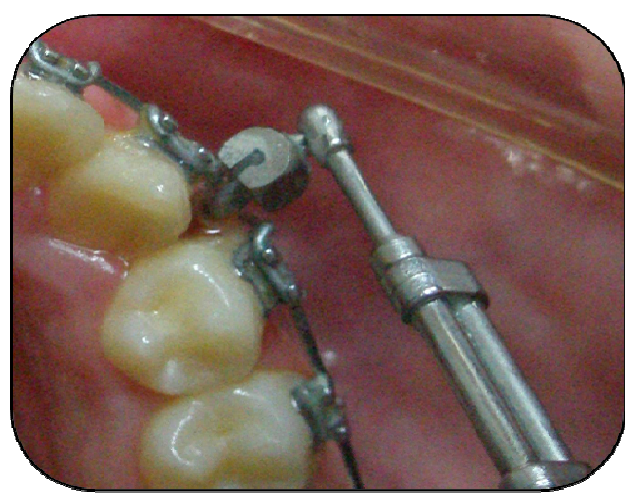

Fig10: Close up view of the TFBC fixed to the miniplate

Volume 40-Decem6er 2011 
Egyptian

Orthodontic Journal

A panoramic x-ray was taken to verify the contact between the miniplate and the bone and the relation with the adjacent teeth. After 2-3 weeks the skeletal anchorage system was loaded with TFBC.

When molars and canines Class I was obtained, the same procedure done for patients of the first group was repeated.

Cephalometric landmarks: (Fig 11)

The following plans were selected:

- SN plane: constructed between $\mathrm{N}$ and $\mathrm{S}$ points representing the anterior cranial base.

- A modified 'Frankfort' horizontal plane (FH) was drawn with an inferior angle of 7 degrees to SN plane through point $\mathrm{S}$

Frankfort horizontal perpendicular (FHp) was drawn as a perpendicular to 'FH' plane through point $\mathrm{S}$. The ' $\mathrm{FH}$ ' plane thus provided a baseline for vertical linear measurements and Flip as a standard for horizontal linear measurements

Concerning angular measurements, nasolabial angle and soft tissue facial convexity were measured before and after treatment. This latter constituted the angle formed by a line connecting the soft tissue nasion to pronasale and another line connecting pronasale to soft tissue pogonion. Viewing linear measurement, the distance between FHp and upper lip, lower lip and soft tissue pogonion was measured before and after treatment. In addition, pre and post-operative distances of upper and lower lips to E line were recorded. The post-operative change in the vertical distance between anterior nasal spine (ANS) and menton (Me) was also detected. 


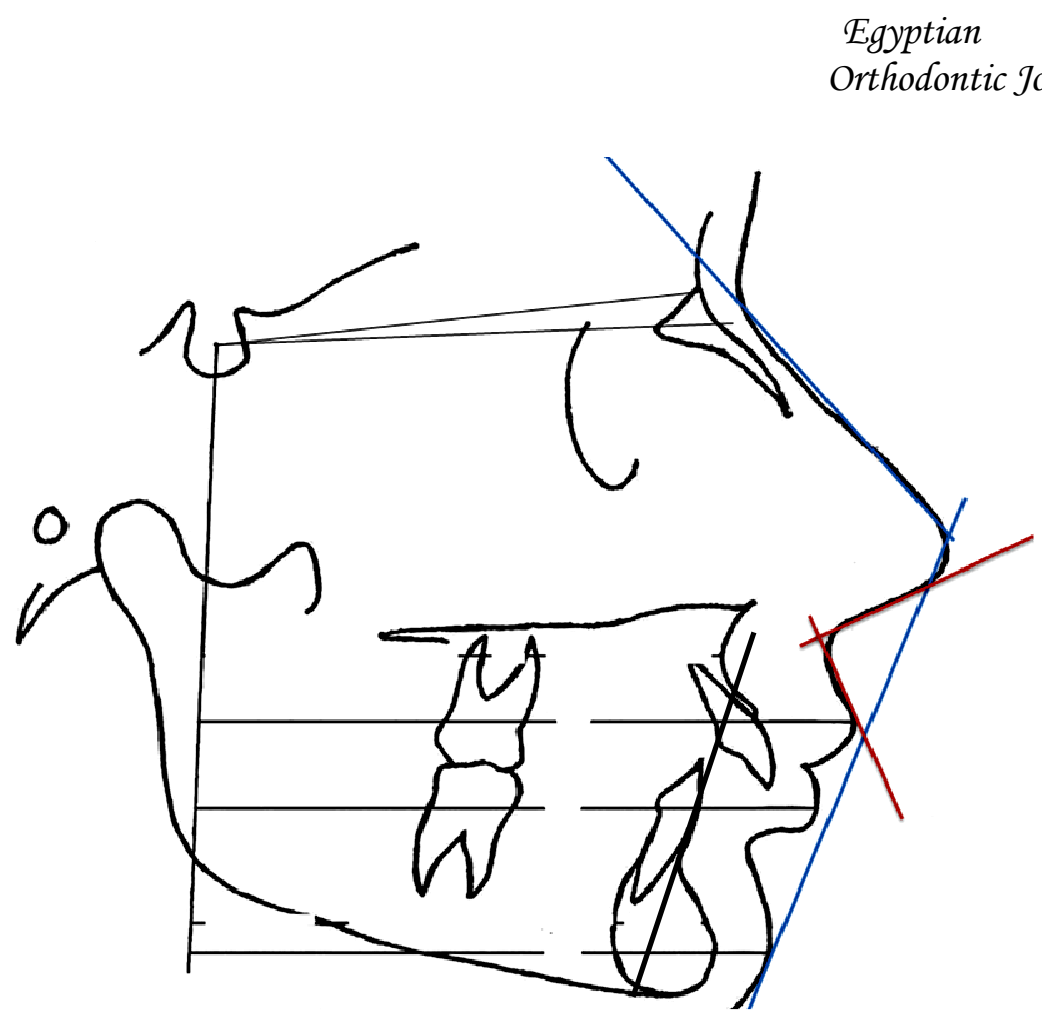

Fig11: Angular and linear measurements taken on the cephalogram

\section{RESULTS}

Group I: (Table 1)

A significant increase was observed in both the soft tissue facial convexity and the nasolabial angle after treatment. (Fig 12) Concerning horizontal measurements (Fig 13), a significant decrease was observed in distance between lower lip and FHp after treatment. This was also observed in the post-operative distance between lower lip and $\mathrm{E}$ line (Fig 14). However, other parameters showed a significant increase after treatment. The vertical line connecting ANS and Me showed a post-operative significant increase. (Fig 15) 
Egyptian

Orthodontic Journal

Table (1): measurement in group I.

\begin{tabular}{|l|c|c|c|}
\hline & Pre-treatment & Post treatment & P \\
\hline Angular measurements (Degrees) & & & \\
\hline Facial convexity & $121.0 \pm 11.2$ & $130.5 \pm 8.22$ & $0.021^{*}$ \\
\hline Nasolabial angle & $80.6 \pm 4.01$ & $90.5 \pm 6.05$ & $0.015^{*}$ \\
\hline Horizontal measurements (mm) & & & \\
\hline UL to FHP & $89.2 \pm 5.12$ & $81.2 \pm 6.21$ & $0.021^{*}$ \\
\hline LL to FHP & $78.3 \pm 4.26$ & $81.9 \pm 4.68$ & $0.31^{*}$ \\
\hline STPog to FHP & $83.1 \pm 3.99$ & $89.5 \pm 3.58$ & $0.0416^{*}$ \\
\hline UL to E line & $-1.1 \pm 0.031$ & $-2.3 \pm 0.106$ & $0.042^{*}$ \\
\hline LL to E line & $-2.5 \pm 0.106$ & $-0.9 \pm 0.036$ & $0.023^{*}$ \\
\hline Vertical Mesurements (mm) & & & \\
\hline ANS-ME & $74.1 \pm 6.01$ & $78.9 \pm 6.46$ & 0.099 \\
\hline
\end{tabular}

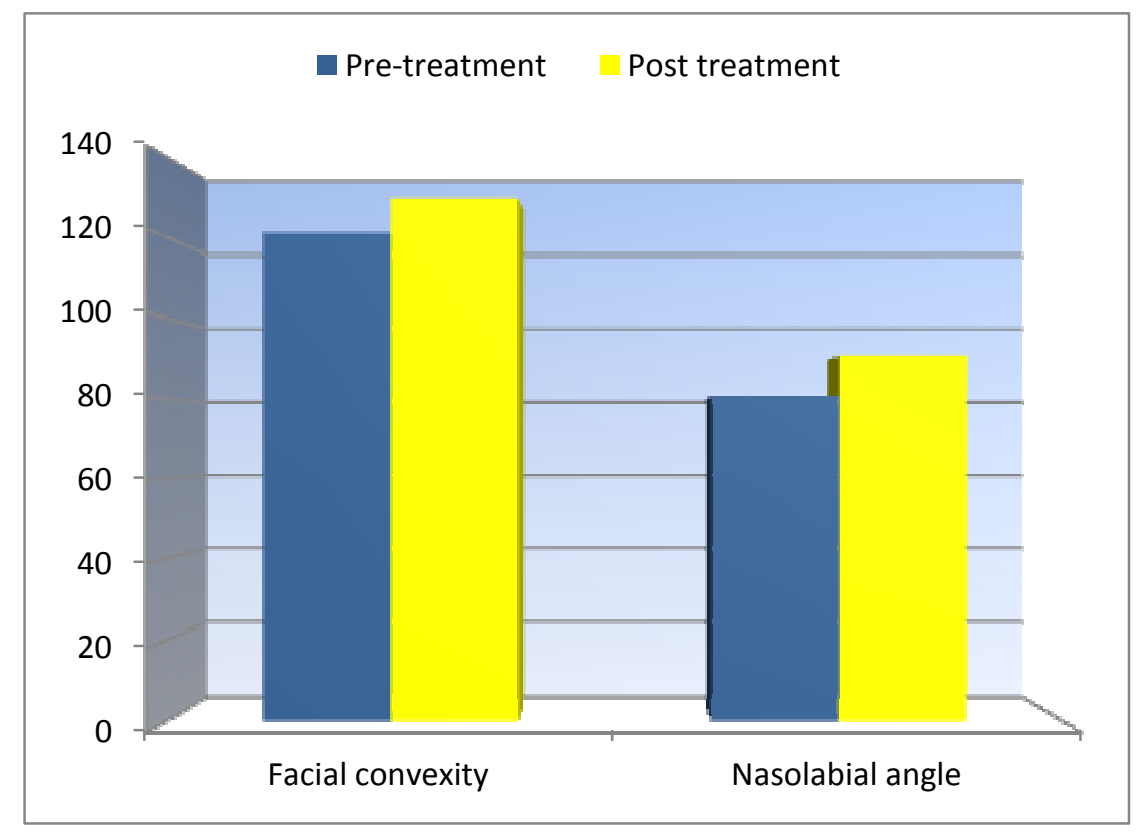

Fig 12: Angular measurements in group 1 


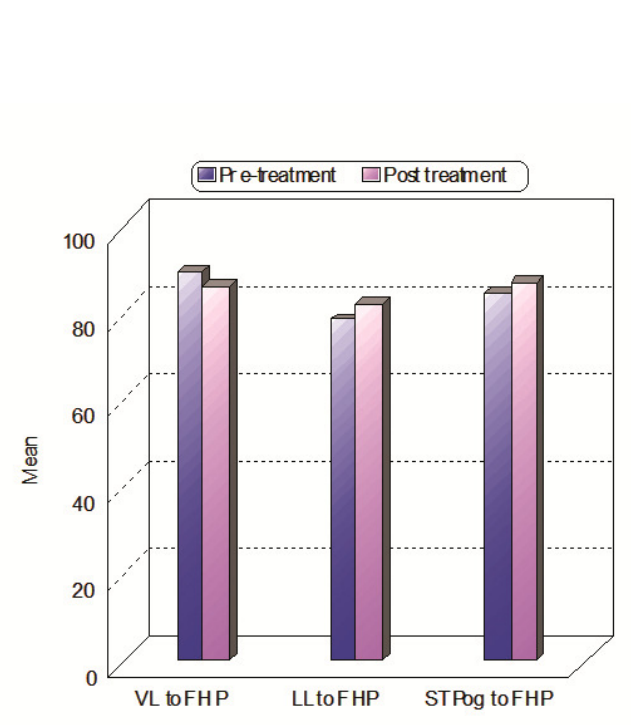

Fig 13 : Linear measurements in group 1
Egyptian

Orthodontic Journal

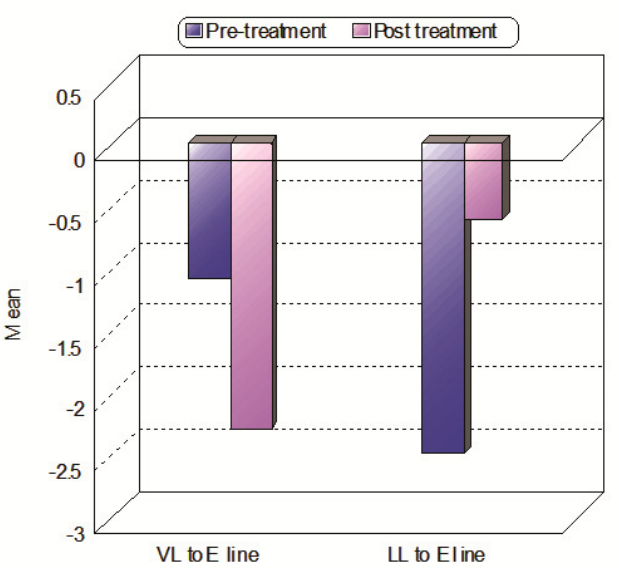

Fig14: Mean difference of upper and Lower lips distances to $\mathrm{E}$ line

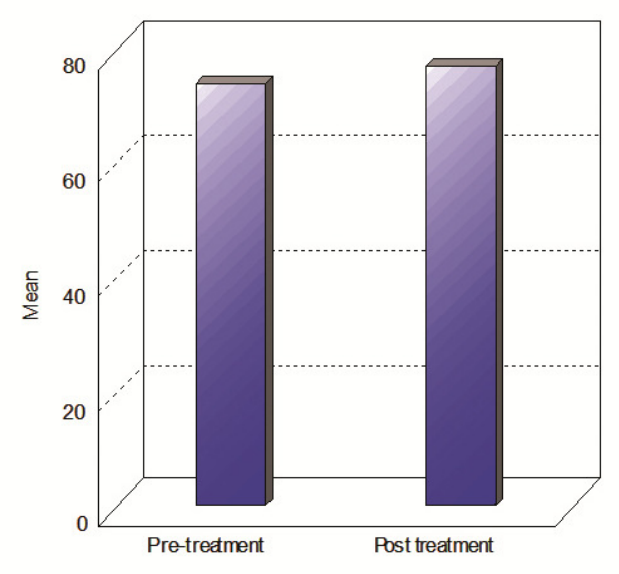

Fig 15: Mean difference in vertical measurements in group 1

Group II: (Table 2)

Like group I, a significant post-operative increase was detected in the naso-labial angle and the soft tissue facial convexity after treatment. (Fig 16) A significant post operative increase was also observed in the distance between FHp and both the lower lip and soft tissue pogonion. However, the distance to the upper lip showed a significant decrease. (Fig 17) Concerning the distance to the E line, the upper lip showed 
a significant increase while the lower lip showed a significant decrease after treatment. (Fig 18) An increase in the distance between ANS and Me was observed. Unlike group I, it was non-significant. (Fig 19)

Table (2): measurement in group II.

\begin{tabular}{|l|c|c|c|}
\hline & Pre-treatment & Post treatment & P \\
\hline Angular measurements (Degrees) & & & \\
\hline Facial convexity & $122.0 \pm 10.3$ & $131.6 \pm 9.01$ & $0.003^{*}$ \\
\hline Nasolabial angle & $81.3 \pm 5.11$ & $91.3 \pm 5.94$ & $0.005^{*}$ \\
\hline Horizontal measurements (mm) & & & \\
\hline UL to FHP & $90.7 \pm 6.02$ & $81.1 \pm 5.85$ & $0.0071^{*}$ \\
\hline LL to FHP & $79.4 \pm 4.11$ & $84.5 \pm 5.29$ & $0.015^{*}$ \\
\hline STPog to FHP & $83.7 \pm 3.82$ & $92.1 \pm 4.28$ & $0.012^{*}$ \\
\hline UL to E line & $-1.21 \pm 0.051$ & $-2.6 \pm 0.106$ & $0.032^{*}$ \\
\hline LL to E line & $-2.46 \pm 0.114$ & $-1.42 \pm 0.011$ & $0.013^{*}$ \\
\hline Vertical Measurements (mm) & & & \\
\hline ANS-ME & $71.2 \pm 6.88$ & $79.8 \pm 5.23$ & $0.039^{*}$ \\
\hline
\end{tabular}

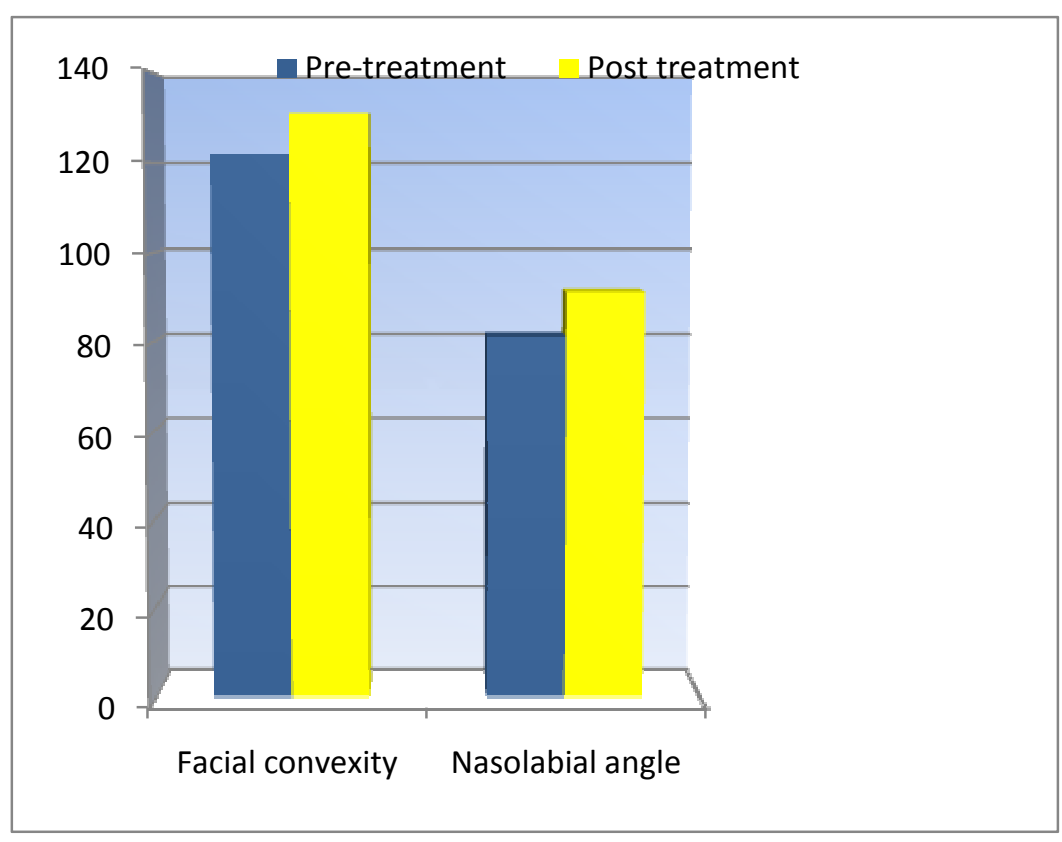

Fig 16: Angular measurements in group 2 

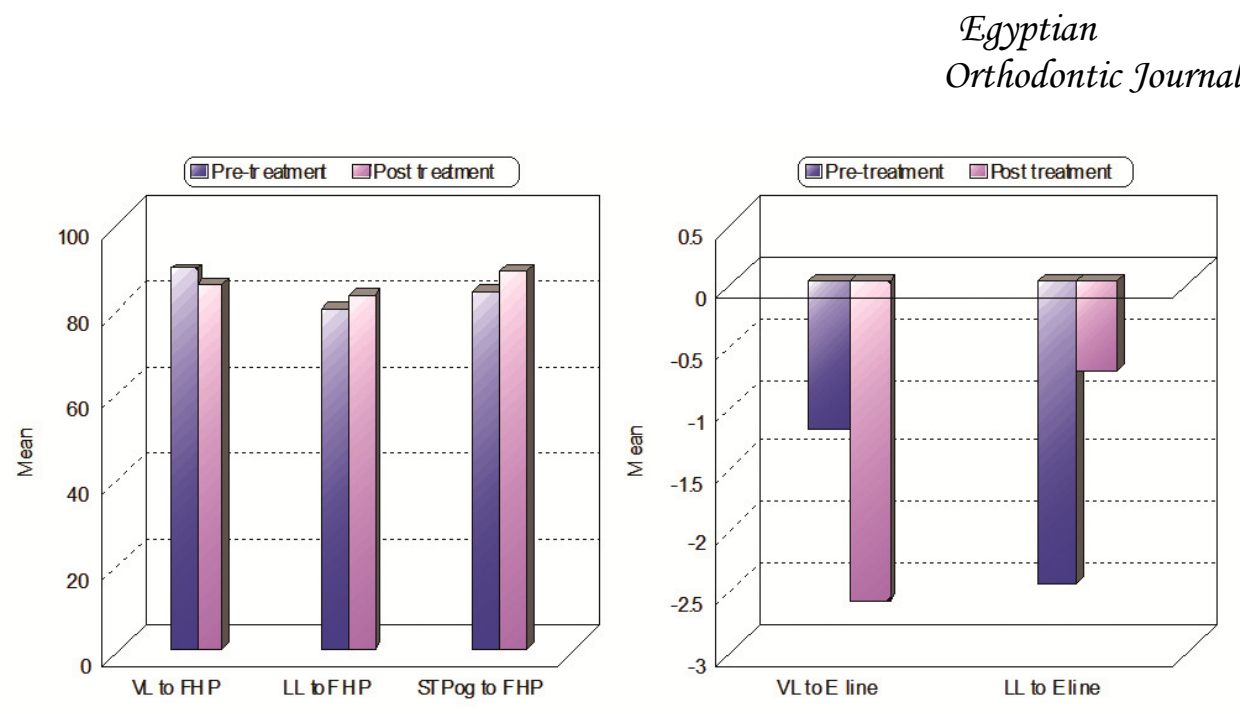

Fig 17: Linear measurements in group 2

Fig 18: Mean difference of upper and lower lips distances to E line

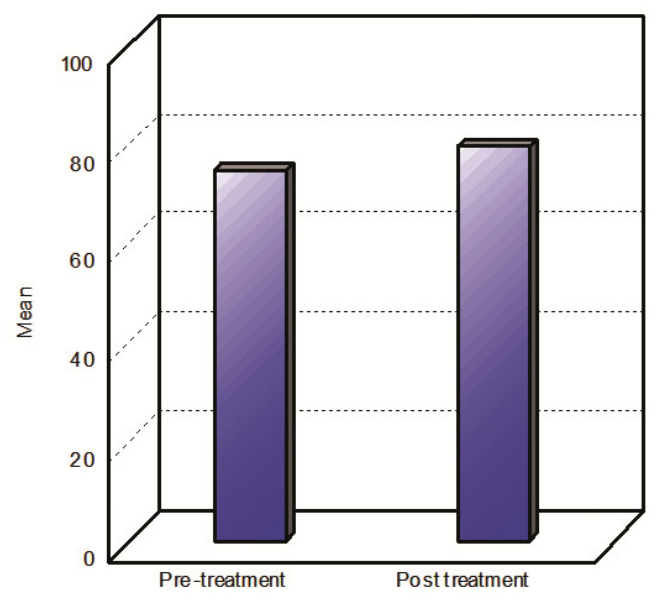

Fig 19: Mean difference in vertical measurements in group 2

Comparison between the results in both groups: (Table 3)

Viewing the angular measurements no statistically significant difference between post-operative results was found. (Fig 20) Concerning horizontal measurements, the only significant difference was found in the distance between the FHp and the soft tissue pogonion. (Fig 21) In 
addition, there was a non significant difference concerning the distance of upper and lower lips to E line in both groups. (Fig 22) There was a significant difference between the results in both groups when considering the distance between ANS and Me post-operatively. (Fig 23)

Table (3): Comparison between group I and II regarding the difference in measurements.

\begin{tabular}{|l|c|c|c|}
\hline & Group I & Group II & P \\
\hline Angular measurements (Degrees) & & & \\
\hline Facial convexity & 9.5 & 9.6 & 0.65 N.S. \\
\hline Nasolabial angle & 9.9 & 10.0 & 0.38 N.S. \\
\hline Horizontal measurements (mm) & & & \\
\hline UL to FHP & -8 & -9.6 & 0.098 N.S. \\
\hline LL to FHP & 3.6 & 5.1 & 0.106 N.S. \\
\hline STPog to FHP & 6.4 & 3.4 & $0.001 *$ \\
\hline UL to E line & -1.2 & -1.39 & 0.085 N.S. \\
\hline LL to E line & 1.6 & 1.04 & 0.32 N.S. \\
\hline Vertical Mesurements (mm) & & & \\
\hline ANS-ME & 8.6 & 4.8 & $0.003 *$ \\
\hline
\end{tabular}

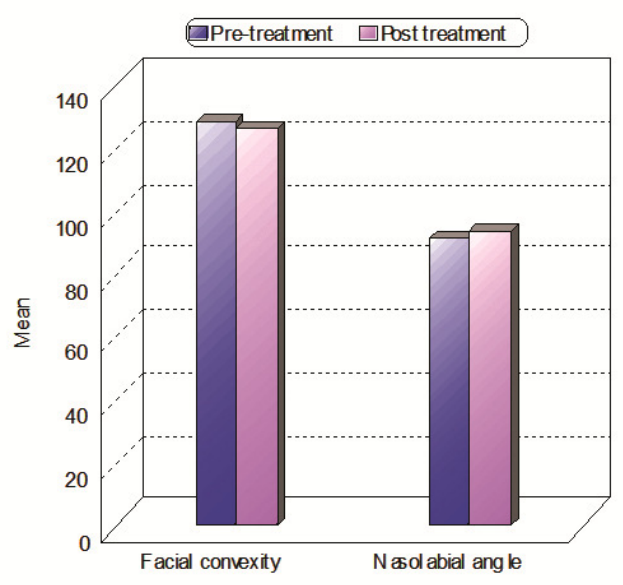

Fig 20 : Comparison between both groups regarding linear measurements

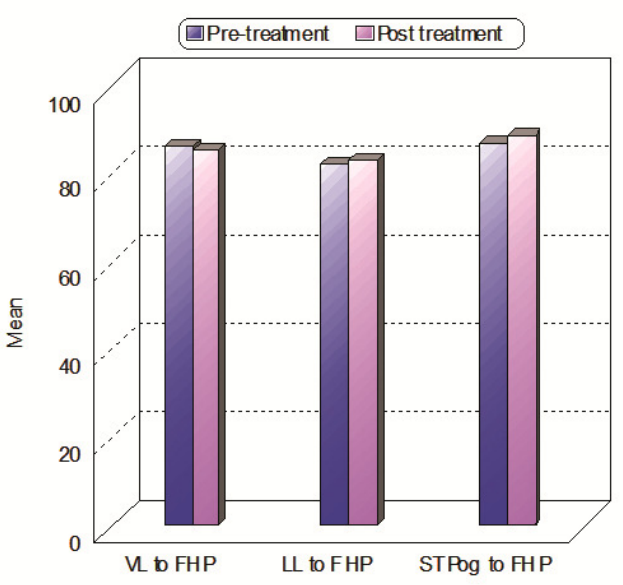

Fig 21: Comparison between both groups regarding angular measurements 


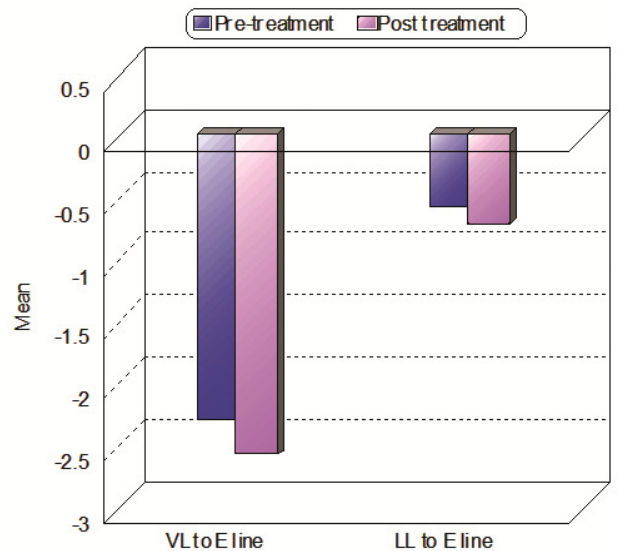

Fig 22: Mean post operative changes in both groups regarding distances of bothlips to $E$ line

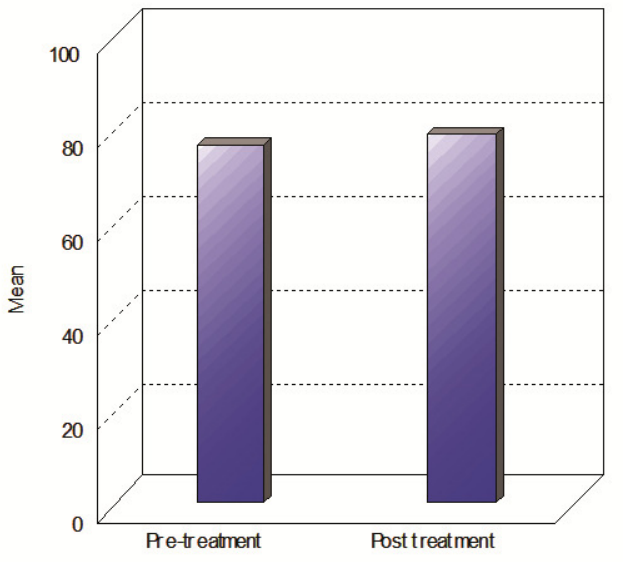

Fig 23: Mean postoperative changes in vertical measurement in both groups

\section{DISCUSSION}

The benefits of using a functional appliance include the growth stimulus, and other more important factors, namely alteration of the profile, smile and facial expressions which helps to improve psychological problems. The fixed functional appliance is indicated for the noncompliance treatment of Class II skeletal discrepancies, mainly in young patients.

The main disadvantages of the fixed functional appliance include anchorage loss of the lower teeth represented by proclination of the lower incisors during treatment (Pancherz and Ruf 2000) ${ }^{(8)}$. In addition, chewing problems during the first week of the treatment, soft tissue impingement, breakage or distortion of the appliance, bent rods, loose or broken bands and in some cases broken or loose screws can be also encountered with.

Titanium miniplates and screws have long been used successfully in maxillofacial surgery for orthognathics, trauma reconstruction, and osseous stabilization. They have shown admirable biocompatibility and predictability when used properly. The application of these surgical devices for orthodontic anchorage is relatively new and has generated much interest in the orthodontic community. ${ }^{(9,10,11)}$ 
That is why this study was designed to use mini-plates as a Temporary Skeletal Anchorage Devices (TSAD) to allow lower bone support to advance the mandible. Therefore, using a skeletal anchorage as miniplate for an orthopedic force from a fixed functional appliance as Twin Force Bite Corrector (TFBC) was a point worthy of investigation

In this study, miniplates offered excellent anchorage over the whole experimental period, withstanding continuous forces of 150 to $200 \mathrm{~g}$. Miniplate placed outside the mandibular dentition functioned as onplants, and the screw functioned as implants, making rigid anchorage possible.

The Twin Force Bite Corrector (TFBC) was introduced by Rothenberg, Campbell and Nanda (2004) ${ }^{(12)}$ as a new fixed intermaxillary functional appliance with a built-in constant force for Class II correction. It was chosen in this study because it is a newly introduced fixed functional appliance with no intensive research on its effects.

The initial age of the patients was set to be 11-14 years to assure being in the early permanent dentition and around the peak of the growth spurt. This was supported by the study of McNamara and colleagues (1985). ${ }^{(4)}$

Moreover, Von Bremen and Pancherz (2002) $)^{(13)}$ found that orthodontic treatment of Class II division 1 started in the permanent dentition was shorter in duration when compared to treatment started at the early or late mixed dentition stages. Another study that supported this age to be suitable for functional appliance therapy was that conducted by Rothenberg, Campbell and Nanda (2004).

The sample of this study included 19 patients. The number of patients in each group was sufficient for statistical purposes in clinical studies (Sas 1998). ${ }^{(14)}$ It was also close to the study of Heinig and Göz (2001). ${ }^{(15)}$ They used a sample of 13 patients to study the effect of the Forsus spring on the treatment of Class II division 1.

Patients were divided into two groups. In the first group, the TFBC was used in the conventional way while in the second group it was used in combination with skeletal anchorage system (SAS)

For patients of both groups pre and post-operative lateral cephalograms were taken. Lateral cephalograms allow the examiner to investigate the different changes produced by functional appliances both 
in the sagittal and vertical planes. They are extensively used in almost every study that evaluated treatment effects of functional appliances. Among those studies were the ones conducted by Weiland et al $(1997)^{(16)}$ and Du, Hägg and Rabie (2002). ${ }^{(17)}$

The design of the miniplate was custom made in order to fulfill the requirement of targeted use. It was an I-shaped plate with three different sections. $1^{\text {st }}$ part endossous section: (15 mm length, $0.7 \mathrm{~mm}$ thickness) with the 3 holes where the miniscrews were screwed in the bone. This was based on the research of Haug $(1993)^{(18)}$ who reported that the increased stability of miniplate fixation is obtained by the increasing the number of screws from 2 to 3 .

Concerning reference plane, a modified 'Frankfort' horizontal plane $(\mathrm{FH})$ was drawn with an inferior angle of 7 degrees to SN plane through point S. This modified FH plane was drawn because it is often difficult to locate Porion accurately, resulting in faulty inclination of the plane and distortion of vertical measurements. Thus, to eliminate possible error and bias, a new FH plane was drawn; which would be more reliable and easily reproducible. This modified FH plane is denoted as 'FH'.

Frankfort horizontal perpendicular (FHp) was drawn as a perpendicular to FH plane through point S. The FH plane thus provided a baseline for vertical linear measurements and FHp as a standard for horizontal linear measurements.

Viewing angular measurements, both the naso-labial angle and soft tissue facial convexity showed a significant increase after treatment. The angle of convexity increased due to mandibular advancement and subsequent increase in the SNB and ANB angles. The results are in accordance with Abdallah (1990). ${ }^{(19)}$

Regarding the TFBC, It was revealed that it had a significant dentoalveolar effects in the form of retroclination of upper incisors and proclination of lower incisors (Aboul Azm 2009). ${ }^{(20)}$ Retroclination of the upper incisors is in turn followed by upper lip retraction which can explain the increase in the naso-labial angle.

This retraction of the upper lip contributed to the decrease in the distance between upper lip and FHp. ${ }^{(21)}$ Since the upper lip was directed backwards, its distance to E line increased. Comparing the results of the two groups, non-significant difference was detected. 
Postoperative mandibular advancement resulted in soft tissue pogonion advancement. Hence the distance between soft tissue pogonion and FHp increased significantly after treatment in both groups. This in turn affected the distance between lower lip and $\mathrm{E}$ which decreased in both groups. However, the decrease was significant the first group. This was attributed to the dento-alveolar effect of TFBC. This was present also when using the skeletal anchorage. The dento-alveolar effect of the fixed functional appliances was proved in various researches. ${ }^{(22,23,24)}$

The increase was significant in the second group. There was also a significant difference between both groups. SAS proved to have minimum effect on the mandibular dentition; while protrusion of lower anterior teeth is detected with TFBC. This might offer a wider range for the mandible to advance downward and forward leading to increase the lower facial height.

\section{CONCLUSIONS}

1- TFBC proved to have a dento-alveolar effect manifested by upper incisors retraction which in turn caused upper lip retraction and increase in the nasolabial angle.

2- Both groups showed improvement of the angle of convexity; hence improvement of the soft tissue profile.

3- There was no statistically significant difference between the results of both groups except the distance of FHp to Pog' and post-operative vertical distance.

4- TAD or SAS cause more mandibular advancement since there is no effect of the protruded lower incisors on the amount of advancement.

\section{REFERENCES}

1- Sahm G, Bartsch A and Witt Eb. Reliability of patient report on compliance. Eur J Orthod 1990; 12:438-446.

2- Sergi HG and Zentner A. Predicting patient compliance in orthodontics. Semin Orthod 2000; 6:231-236. 
3- Nanda RS and Kierl MJ. Prediction of cooperation in orthodontic treatment. Am J Orthod Dentofacial Orthop 1992; 102:15-21.

4- Goldstein MS, Stanton FL. Various types of occlusion and amounts of overbite in normal and abnormal occlusion between two and twelve years. Int J Orthod 1936; 22:549-69. (Cited from Bishara SE. Mandibular changes in persons with untreated and treated Class II division 1 malocclusion.

5- Fisk RO. When malocclusions concern the public. J Can Dent Assoc 1960; 26:397-412.

6- Ast DB, Carlos JP, Cons DC. Prevalence and characteristics of malocclusion among senior high school students in up-state New York. Am J Orthod 1965; 51:437-45.

7- McNamara JA. Components of Class II malocclusion in children 810 years of age. Angle Orthod 1981; 51:177-202.

8- Pancherz H, Ruf S. The Herbst appliance: research-based updated clinical possibilities. World J Orthod 2000; 1:17-31

9- Umemori M, Sugawara J, Mitani H, Nagasaka H, Kawamura H. Skeletal anchorage system for open- bite correction. Am J Orthod Dentofacial Orthop 1999; 115:166-174.

10- Sherwood KH, Burch JG. Skeletally based miniplate supported orthodontic anchorage. J Oral Maxillofac Surg 2005; 63: 279- 284.

11- Londa G. The anchorage quality of titanium microplates with short microscrews for orthodontic anchorage applications. J Orofac Orthop 2005; 66:67-77.

12- Rothenberg J, Campbell ES and Nanda R. Class II correction with the twin force bite corrector. JCO 2004; 38(4):232-240.

13- Von Bremen J and Pancherz H: Efficiency of Class II, division 1 and Class II division 2 treatment in relation to different treatment approaches. Semin Orthod. 2003;9: 87-92.

14- SAS (1988). Statistical Analysis system, STAT/User's Guide, Release 6.03 ed, SAS Institute, Cary NC, USA. 
15- Heinig N and Goz G: Clinical application and effect of the Forsus spring. A study of a new Herbst hybrid. J orofac Orthop. 2001;62: 436-450

16- Weiland FJ, Ingervall B, Bantleon HP and Drochat H. Initial effects of treatment of Class II malocclusion of Herren activator. Activator-head gear combination, and Jasper Jumper. Am J Orthod Dentofac Orthop. 1997; 112:19-27.

17- Du X, Hagg EU, and Rabie ABM: Effect of head gear Herbst and mandibular step-by-step advancement versus conventional Herbst appliance and maximal Jumping of the mandible. Eur J Orthod 2002;24: 167-174.

18- Haug R: The effect of screw number and length on two methods of tension and plating. J oral Maxillofac Surg 1993; 51-62.

19- Abdallah MG: A comparison between Herbst appliance and activator in treatment of Class II, division 1 malocclusion. PhD thesis, Cairo University, 1990.

20- Aboul Azm KS: Evaluation of the efficacy of the fixed functional appliance supported with skeletal anchorage system for treatment of developing Class II malocclusion. PhD thesis, Minia University, 2009.

21- Refai WM: Changes in the lip in position and function during orthodontic treatment. PhD thesis, Cairo University, 2002.

22- Weschler D and Pancherz H. Efficiency of three mandibular anchorage forms in Herbst treatment: A cephalometric investigation. Angle Orthod. 2005; 75(1): 23-27.

23- De Almeida MR, Henriques JFC, De Almeida RR, Ursi W and McNamara JA Jr: Short-term treatment effects produced by the Herbst appliance in the mixed dentition. Angle Orthod. 2005; 75(4): 540-547.

24- Lisson JA and Trankmann J. Treatment begin and treatment effect in functional orthopedics with jumping-the-bite appliances. J Orofac Orthop 2005; 66:67-77. 